\title{
Produção de massa verde e ganho em peso de peixes ornamentais em mini sistema doméstico de aquaponia
}

\section{Green pasta production and weight gain of ornamental fish in a mini domestic aquaponics system}

Wesley Clovis Barbieri Mendonça Universidade Federal da Grande Dourados

E-mail: wesley.clovis@hotmail.com

OrcID: https://orcid.org/0000-0001-9994-8966

Anderson Ferreira Santana

Universidade Federal da Grande Dourados

E-mail: andersonferreirasantana@gmail.com

OrclD: https://orcid.org/0000-0001-8609-536X

Arypes Scuteri Marcondes

Universidade Federal da Grande Dourados

E-mail: arypes@hotmail.com

OrcID: https://orcid.org/0000-0003-0384-0104

David Geovanni de Almeida Banhara

Universidade Federal da Grande Dourados

E-mail: davidgalmeida@outlook.com

OrcID: https://orcid.org/0000-0002-4614-2050

Rebeca Maria Sousa

Universidade Federal de Mato Grosso do Sul

E-mail: rebeca_sousa31@hotmail.com

OrcID: https://orcid.org/0000-0002-1195-3304

Henrique Momo Ziemniczak

Universidade Federal da Grande Dourados

E-mail: henrique.momo1@gmail.com

OrcID: https://orcid.org/0000-0002-7198-8939

Luis Antonio Kiosh Oaki Inoue

Embrapa Agropecuária Oeste

E-mail: luis.inoue@embrapa.br

OrclD: https://orcid.org/0000-0002-9582-7654

Claucia Aparecida Honorato da Silva

Universidade Federal da Grande Dourados

E-mail: clauciahonorato@ufgd.edu.br

OrcID: https://orcid.org/0000-0002-7161-4996

Resumo: A aquaponia é a integração da aquicultura com a produção vegetal, onde os resíduos da primeira atividade servem de insumos para a segunda, por meio de transformações biológicas que ocorrem no sistema. Este trabalho teve como objetivo avaliar o efeito da densidade inicial de peixes ornamentais (Carassius auratus) em mini sistemas de aquaponia, compostos por aquários de $100 \mathrm{~L}$ e camas de cultivo com argila expandida $(20 \mathrm{~L})$. Foram avaliadas duas densidades (três e seis peixes por sistema), com três repetições. Cada cama de cultivo recebeu uma muda de manjericão (Ocimium basilicum) e uma de salsinha (Petroselinum crispum). Os peixes foram alimentados diariamente até a saciedade aparente com ração comercial de $32 \% \mathrm{~PB}$, duas a três vezes por dia durante 103 dias. Os valores mais altos de ganho em peso 
dos peixes foram observados nos aquários com menor densidade. A produção de massa verde foi mais elevada nos sistemas de maior densidade inicial de peixes. Conclui-se que a maior densidade inicial de peixes proporcionou melhor produção de massa verde, apesar de apresentar menores índices de crescimento dos peixes.

Palavras-chave: Carassius auratus; Densidade de estocagem; Kinguio; Sustentabilidade.

Abstract: Aquaponics is the integration of aquaculture with crop production, where the residues of the first activity serve as inputs for the second, through biological transformations that occur in the system. The objective of this work was to evaluate the effect of the initial density of ornamental fish (Carassius auratus) in mini aquaponics systems, consisting of $100 \mathrm{~L}$ aquariums and expanded clay cultivation beds (20 L). Two densities (three and six fish per system) were evaluated with three replications. Each growing bed received one seedling of basil (Ocimium basilicum) and one of parsley (Petroselinum crispum). The fish were fed daily to apparent satiety with $32 \%$ CP commercial feed, two to three times daily for 103 days. The highest values of fish weight gain were observed in lower density aquariums. Green mass production was higher in systems with higher initial fish density. It was concluded that the higher initial density of fish provided better production of green mass, despite presenting lower fish growth rates.

Keywords: Carassius auratus; Stocking density; Kinguio; Sustainability.

Data de recebimento: $26 / 03 / 2020$

Data de aprovação: $27 / 07 / 2020$

DOI: https://doi.org/10.30612/agrarian.v13i50.11300

\section{Introdução}

A palavra "aquaponia" é a combinação entre "aquicultura" (produção de organismos aquáticos) e "hidroponia" (produção de plantas sem solo) e em suma é a junção destas duas (CARNEIRO et al., 2015). Neste sistema de produção os peixes são mantidos e alimentados em sistemas fechados, onde a água após passar por filtragem mecânica e biológica retorna ao sistema (RAHMATULLAH et al., 2010).

A aquaponia é um sistema de produção de alimento que articula a aquicultura e a horticultura em sistemas de recirculação de água e nutrientes (HUNDLEY \& NAVARRO, 2013). Esta modalidade é uma opção para a produção de alimentos de forma sustentável com diminuição dos impactos ambientais pelas características de sustentabilidade (CELESTRINO \& VIEIRA, 2018). Dentro do contexto de economia cíclica e de sustentabilidade, pequenos produtores podem produzir peixes e hortaliças utilizando-se pequenos recipientes (DIVER, 2006).

A produção integrada de peixes e vegetais vem chamando a atenção em muitos países do mundo, especialmente em iniciativas da agricultura urbana que tem por finalidade estimular as pessoas a produzirem o próprio alimento ou pelo menos parte deste em espaços reduzidos (BRANCO \& ALCÂNTARA, 2011; CARNEIRO et al., 2015). Nesse sentido, a aquaponia vem sendo empregada em diversas escalas, desde grandes projetos industriais como os já instalados nos Estados Unidos até unidades domésticas pequenas. Porém, é um sistema que carece de maiores informações técnico-científicas como os efeitos da densidade inicial de peixes, a possibilidade de uso de peixes ornamentais ou ainda quais as limitações do uso desse tipo de animal aquático (HUSSAIN et al., 2015).

Dentre os peixes ornamentais que se destacam no sistema de produção está o kinguio do gênero Carassius, pela docilidade e pela rusticidade diante das condições de confinamento em aquários (SIQUEIRA et al., 2018). A utilização de peixes ornamentais para o uso de aquaponia pode ser um atrativo principalmente para as hortas urbanas, uma vez que este peixe poderá ser comercializado por ter maior valor agregado.

As políticas públicas de incentivo a hortas urbanas e periurbanas impulsionaram o desenvolvimento dessa estratégia de combate à pobreza (BRANCO \& ALCÂNTARA, 2011), com o incentivo de alimentação mais saudável. Dentre os produtos de predileção das casas urbanas na sua produção estão os condimentos como: salsinha, cebolinha (ZÁRATE et al., 2006).

A utilização deste sistema pode ser realizada em ambientes domésticos enfatizando a educação ambiental associada à qualidade alimentar das famílias. Neste contexto, o uso de condimentos neste sistema 
é uma alternativa para ser utilizada em ambientes urbanos uma vez que há uma demanda crescente por alimentos saudáveis com procedência conhecida (CARRILHO et al.,2017).

Assim, foi testado neste trabalho os efeitos da densidade inicial de peixes ornamentais, kinguio, em mini sistema de aquaponia recém-instalados. Os dados gerados contribuem para o avanço do conhecimento e manejo da aquaponia, bem como auxiliam no fortalecimento da aquaponia em escala doméstica.

\section{Material e Métodos}

\section{Minis sistemas de aquaponia}

Foram utilizados seis minis sistemas de aquaponia. Cada sistema era composto por uma caixa plástica de $100 \mathrm{~L}(75 \times 40 \times 55 \mathrm{~cm})$, uma bomba submersa de aquário $(500 \mathrm{~L} / \mathrm{h})$ e uma cama de cultivo com argila expandida (20 L), alojadas em caixa plástica adaptadas $(55 \times 35 \times 15 \mathrm{~cm})$, de forma a proporcionar a circulação da água (Figura 1) Os dados de temperatura para Dourados durante o experimento foram acompanhados pelo site Guia Clima - Embrapa Agropecuária Oeste (FIETZ, 2020)

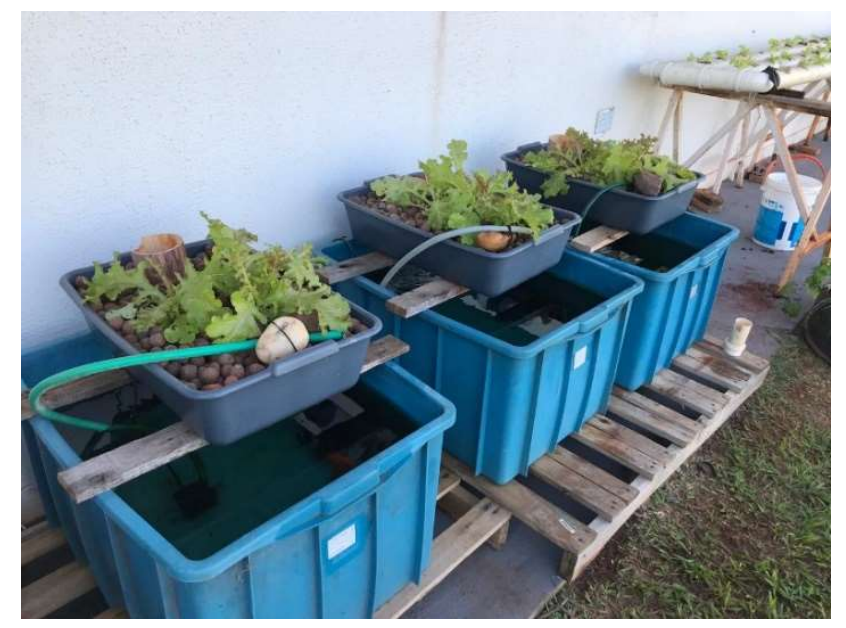

Figura 1. Composição do mini sistema aquapônico

\section{Instalação e rotina do experimento}

Foram utilizados dois tratamentos, o primeiro com 3 peixes e o segundo com 6 peixes por sistema, em triplicata. Os peixes utilizados foram da espécie Carassius auratus (Figura 2). Onde foram distribuídos de forma aleatória e alimentados de três vezes ao dia até saciedade aparente, com ração comercial (36\% PB), por 103 dias. O experimento foi realizado entre os meses de janeiro a abril de 2019, quando as temperaturas estão mais elevadas na região de Dourados-MS, chegando a média de $31,6^{\circ} \mathrm{C}$ durante o período experimental (GUIA CLIMA, 2019).

Cada cama de cultivo recebeu uma muda de salsinha e outra de manjericão, retiradas de bandejas de produção de mudas, as quais apresentavam plantas homogêneas em tamanho. Semanalmente as caixas eram sifonadas até a metade para limpeza e a água reposta.

Foram avaliados a massa verde das mudas e o crescimento dos peixes. A massa verde foi pesada no final do experimento logo após o corte na base das plantas. Os peixes foram contados, pesados e medidos no início e no final do experimento.

\section{Cálculos e análises de dados}

A partir dos valores de peso e comprimento dos peixes no início e fim do experimento em cada mini sistema de aquaponia foram calculados:

- Biomassa inicial $(\mathrm{g} / \mathrm{caixa})=$ somatório do peso individual no início do experimento

- Biomassa final $(\mathrm{g} / \mathrm{caixa})=$ somatório peso individual no final do experimento

- Ganho de biomassa (g/caixa) = Biomassa final (g/caixa) - Biomassa inicial (g/caixa) 
- Ganho em peso $(\mathrm{g})$ = Peso médio final $(\mathrm{g})$ - Peso médio inicial $(\mathrm{g})$

- Ganho em comprimento $(\mathrm{cm})=$ Comprimento médio final $(\mathrm{cm})$ - Comprimento médio inicial $(\mathrm{cm})$.

- Sobrevivência $=$ (número de peixes no final do experimento/densidade inicial) *100

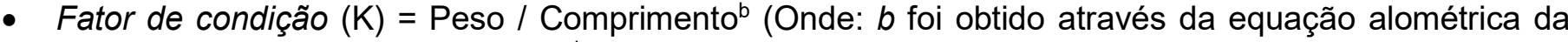
relação peso / comprimento $\left(y=a x^{b}\right)$.

Os dados foram submetidos ao teste $U$, não paramétrico de Mann-Whitney para a constatação diferença estatística ao nível de $95 \%$.

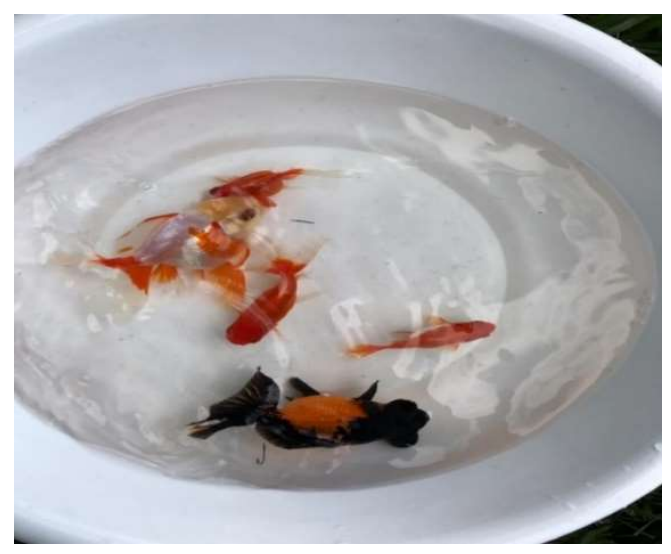

Figura 2. Peixes utilizados nos minis sistema aquapônico

\section{Resultados}

O ganho em peso dos peixes e fator de condição foi maior com 3 peixes por caixa (Tabela 1). Os valores de peso e comprimento apresentaram variações amplas já no início do experimento, devido à dificuldade de padronização inicial dos peixes, fato comum ao se trabalhar com uma espécie de peixe ornamental como o kinguio (Carassius auratus). O ganho de biomassa e ganho em comprimento aparentemente foram melhores com 3 peixes por caixa, mas não houve diferença.

Tabela 1. Efeito da densidade de kinguios (Carassius auratus) na aquaponia sobre os organismos aquáticos e massa verde do manjericão (Ocimum basilicum) e salsinha (Petroselinum crispum).

\begin{tabular}{lll}
\hline Variáveis & Número de peixes/sistema & \\
\cline { 2 - 3 } & $\mathbf{3}$ & $\mathbf{6}$ \\
\hline Biomassa inicial $(\mathrm{g} / \mathrm{cx})$ & $77,5 \pm 35,7$ & $97,1 \pm 32,2$ \\
Biomassa final $(\mathrm{g} / \mathrm{cx})$ & $135,5 \pm 35,7$ & $142,0 \pm 37,6$ \\
Ganho de biomassa $(\mathrm{g})$ & $58,1 \pm 5,5$ & $44,8 \pm 15,3$ \\
Peso inicial $(\mathrm{g})$ & $25,8 \pm 12,3$ & $16,2 \pm 5,3$ \\
Peso final $(\mathrm{g})$ & $45,2 \pm 11,9$ & $28,1 \pm 2,0$ \\
Ganho em peso $(\mathrm{g})$ & $19,4 \pm 1,8^{\mathrm{a}}$ & $11,9 \pm 3,9^{\mathrm{b}}$ \\
Comprimento inicial $(\mathrm{cm})$ & $11,3 \pm 2,2$ & $8,2 \pm 0,7$ \\
Comprimento final $(\mathrm{cm})$ & $14,0 \pm 1,8$ & $9,6 \pm 0,1$ \\
Ganho em comprimento $(\mathrm{cm})$ & $2,7 \pm 0,8$ & $1,4 \pm 0,9$ \\
Sobrevivência $(\%)$ & 100 & $83 \pm 17$ \\
Fator de condição & 5,52 & 3,28 \\
Massa verde $(\mathrm{g})$ & $10,8 \pm 12,8^{\mathrm{a}}$ & $73,9 \pm 50,3^{\mathrm{b}}$ \\
\hline
\end{tabular}

*Médias seguidas de letras distintas na mesma linha diferem pelo teste Tukey $(p<0,05)$

A produção de massa verde (Figura 3) apresentou-se pouco vigorosa em todos os sistemas de aquaponia até aproximadamente 90 dias, provavelmente, devido ao calor intenso do verão na região de Dourados, MS, além da recente instalação dos sistemas. Ao final do experimento, os valores de massa verde foram mais altos nos minis sistemas de aquaponia mantidos com seis peixes. 


\section{Referências}

AZAD, A.K.; ISHIKAWA, K.; DIAZ-PEREZ, J.C.; EATON, T.E.; TAKEDA, E.N. Growth and development of komatsuna (Brassica rapa L. Nothovar) in NFT (nutrient film technique) system, as influenced by natural mineral. Agricultural Sciences, v. 4, p. 1-7, 2013.

HEREDIA ZÁRATE, N.A.; VIEIRA, M.C.; GRACIANO, J.D.; HELMICH, M; GASSI, R.P.; SOUZA, C.M. Produção do taro 'Chinês' em cultivo solteiro e consorciado com chicória. Ciência e Agrotecnologia, v. 31, n. 5, p. 1558-1562, 2007.

BALDWIN, L. The effects of stocking density on fish welfare. The Plymouth Student Scientist, v. 4, n. 1, p. 372-383, 2010.

CALÓ, P. Introducción a la Acuaponia. Centro Nacional de Desarrollo acuícola (CENADAC). Ministerio de Agricultura, Ganaderia y Pesca, 2011. 15 p.

CARNEIRO, P.C.F.; MORAIS, C.A.R.S.; NUNES, M.U.C.; MARIA, N.A; FUJIMTO, R.Y. Produção integrada de peixes e vegetais em aquaponia. Aracaju: Embrapa Tabuleiros Costeiros, Comunicado Técnico, 189, 2015. 23 p.

CASTELO, B.M.; ALCÂNTARA, F.A. Hortas urbanas e periurbanas: o que nos diz a literatura brasileira? Horticultura Brasileira, v. 29, p. 421-428, 2011.

DELAIDE, B.; DELHAYEA, G.; DERMIENCE, M.; GOTT, J.; SOYEURT, H.; JIJAKLI, H. Plant and fish production performance, nutrient mass balances, energy and water use of the PAFF Box, a small-scale aquaponic system. Aquacultural Engineering, v. 78, p. 130-39. 2017.

DIVER, S. Aquaponics: integration of hydroponics with aquaculture. Butte: National Sustainable Agriculture Information Service, p. 1-28, 2006.

ENDUT, A.; LANANAN, F.; HAJAR, S.; HAMID, A.; JUSOH, A.; NIK, W.N.W. Balancing of nutrient uptake by water spinach (Ipomoea aquatica) and mustard green (Brassica juncea) with nutrient production by African catfish (Clarias gariepinus) in scaling aquaponic recirculation system. Desalination and Water Treatment, v. 57, n. 60, p. 29531-29540, 2016.

FIETZ, C.R. Guia Clima, Boltetim Agrometereológico - Embrapa Agropecuária Oeste, Edição: março de 2020. Dourados, 2020.

Disponível

em: https://clima.cpao.embrapa.br/?lc=site/boletins/boletinsDetalhes\&ci=143\&cat=Temperatura\&edicao=Edi\%C3\% A7\%C3\%A3o:\%20Mar\%C3\%A70\%20de\%202020\&mes=Mar\%C3\%A7o\#detalheBoletim

GEISENHOFF, L.O.; JORDAN, R.A.; SANTOS, R.C.; OLIVEIRA, F.C.; GOMES, E.P. Effect of different substrates in aquaponic lettuce production associated with intensive tilapia farming with water recirculation system. Engenharia Agrícola, v. 36, p. 291- 299, 2016.

GUIA CLIMA. 2019. Embrapa Agropecuária Oeste. Disponível em: < https://clima.cpao.embrapa.br/?lc=site/boletins/pesquisa>. Acesso em 07 de setembro de 2020.

GRABER, A.; JUNGE, R. 2009. Aquaponic systems: Nutrient recycling from fish waste water by vegetable production. Desalination, v. 246, n. 1-3, p. 147-156, 2009.

HONORATO, C.A.; TESSER, M.B.; PORTELLA, M.C.; CARNEIRO, D.J. Microdietas na alimentação da tilapia do nilo durante a fase de reversão sexual. Nucleus Animalium, v.4, n.1, p. 28-36, 2012.

HUSSAIN, T.; VERMA, A.K.; TIWARI, V.K.; PRAKASH, C.; RATHORE, G.; SHETE, A.P.; SARAHRAN, N. Effect of water flow rates on growth of Cyprinus carpio and spinach plant in aquponic system. Aquaculture International, v. 23, n. 1, p. 369-384, 2015.

INOUE, L.A.K.A.; BEZERRA, A.C.; MIRANDA, W.S.; MUNIZ, A.W.; BOIJINK, C.L. Cultivo de tambaqui em gaiolas de baixo volume: efeito da densidade de estocagem na produção de biomassa. Ciência Animal Brasileira, v. 15, n. 4, p. 437-443, 2014.

LOVE, D.C.; FRY, J.P.; GENELLO, L.; HILL, E.S.; FREDERICK, J.A.; LI, X.; SEMMENS, K. An international survey of aquaponics practitioners. PloS one, v. 9, n. 7, p. e102662, 2014. 
MACIEL, E.; FEITOSA, K.C.; CORREAA-NETO, C.R.; MACEDO, F.; MATTIOLI, W.; ABIMORAD, E.; ABREU, J. Desempenho produtivo e parâmetros fisiológicos de juvenis de pacu criados em tanques-rede em diferentes densidades de estocagem. Revista Brasileira de Saúde e Produção Animal, v. 14, n. 1, p. 185194, 2013.

NUWANSI, K.K.T; VERMA, A.K; TIWARI, V.K; PRAKSH, C.; CHANDRAKAN, M.H. Satandartization of the stocking density ratios of Koi (Cyprinos carpio var: Koi): Goldifish (Cassius auratus) in polycuture Aquaponic Recirculation System, Turkish Journal of Fisheries and Aquatic Sience, v. 17, n. 6, p. 271-1278, 2017.

RAHMATULLAH, R.; DAS, M.; RAHMATULLAH, S.M. Suitable stocking density of tilapia in an aquaponic system. Bangladesh Journal of Fisheries Research, v. 14, n. 1-2, p. 29-35, 2010.

ROSSI-WONGSTSCHOWKI, C.L.D.B. Estudo das variações da relação peso total/comprimento total e função do ciclo reprodutivo e comportamento de Sardinella brasiliensis (Steindachner, 1879) da costa do Brasil entre $23^{\circ} \mathrm{S}$ e $28^{\circ} \mathrm{S}$. Boletim Instituto Oceanográfico, v. 26, p. 131-180, 1977.

SAUFIE, S.; ESTIM, A.; TAMIN, M.; HARUN, A.; OBONG, S.; MUSTAFA, S. Growth performance of tomato plant and genetically improved farmed tilapia in combined aquaponic systems. Asian Journal of Agricultural Research, v. 9, n. 3, p. 95-103, 2015.

SHETE, A.P.; VERMA, A.K.; TANDEL, R.S.; Prakash, C.; Tiwari1, V. K; Hussain, T. Optimization of water circulation period for the culture of goldfish with spinach in aquaponic system. Journal of Agricultural Science, v. 5, n. 4, p. 26, 2013.

SHETE, A.P; VERMA, A.K; KOHLI, M.P.S.; DASH, A.; TANDEL, R. Optimum stocking density for growth of goldfish, Carassius auratus (Linnaeus, 1758), in an aquaponic system. Israeli Journal of AquacultureBamidgeh, v. 65, p. 1-6, 2013.

SUMMERFELT, S.T.; VINCI, B. J. Avoiding water quality failures: Part 1 - Carrying capacity and water flow in intensive aquaculture systems. World aquaculture, v. 35, n. 4, p. 6-8, 2004.

VALLADAO, G.M.R.; GALLANI, S.U.; PILARSKI, F. Phytoterapy as an alternative for treating fish disease. Journal of veterinary pharmacology and therapeutics, v. 38, n. 5, p. 417-428, 2015.

WEATHERLEY, A.H.; GILL, H.S. The biology of fish growth. Academic Press, 1987. 443p. 